\title{
Improving the energy method for calculating beams with a narrow rectangular section on the side buckling
}

\author{
Serdar Yazyev ${ }^{1}$, Anastasia Lapina ${ }^{1}$, Ivan Zotov $^{1}$, Anton Chepurnenko ${ }^{1, *}$, and Irina \\ Doronkina $^{2}$ \\ ${ }^{1}$ Don State Technical University, 1 Gagarin sq., Rostov-on-Don, 344000, Russia \\ ${ }^{2}$ Russian State Tourism and Service University, 99 Glavnaya street, Moscow, 141221, Russia
}

\begin{abstract}
We propose an improved version of the energy method in calculating rectangular beams for the stability of a flat bending shape. The essence of this variant of the method is to use the principle of the minimum total energy instead of the condition for the equality of the potential strain energy and the work of external forces. This version of the method makes it easy to obtain a numerical-analytical solution for any number of members of series. The solution of the problem for a pivotally supported beam is presented taking into account the vertical displacement of the load relative to the center of gravity.
\end{abstract}

\section{Introduction}

For the first time, the energy method for calculating the stability of a flat bending shape of beams was applied by S.P. Tymoshenko [1]. A variant of the method proposed by S.P. Tymoshenko involves determining the critical load from the condition that the work of the external forces is equal to the potential energy of the lateral bending and torsion of the beam. The potential strain energy is expressed in terms of the twist angle of the beam and then the function of the twist angle is found in the form of a trigonometric series. With one member of the series, the problem reduces to a linear equation [2]. In the case of $n$ members of the series, we will have a nonlinear equation of order $n$.

We will propose a variant of the energy method that allows us to obtain a solution for any number of members of the series.

\section{Methods}

The calculation method will be demonstrated by the example of a pivotally supported beam under the action of a load uniformly distributed along the length (Fig. 1).

\footnotetext{
${ }^{*}$ Corresponding author: anton chepurnenk@mail.ru
} 


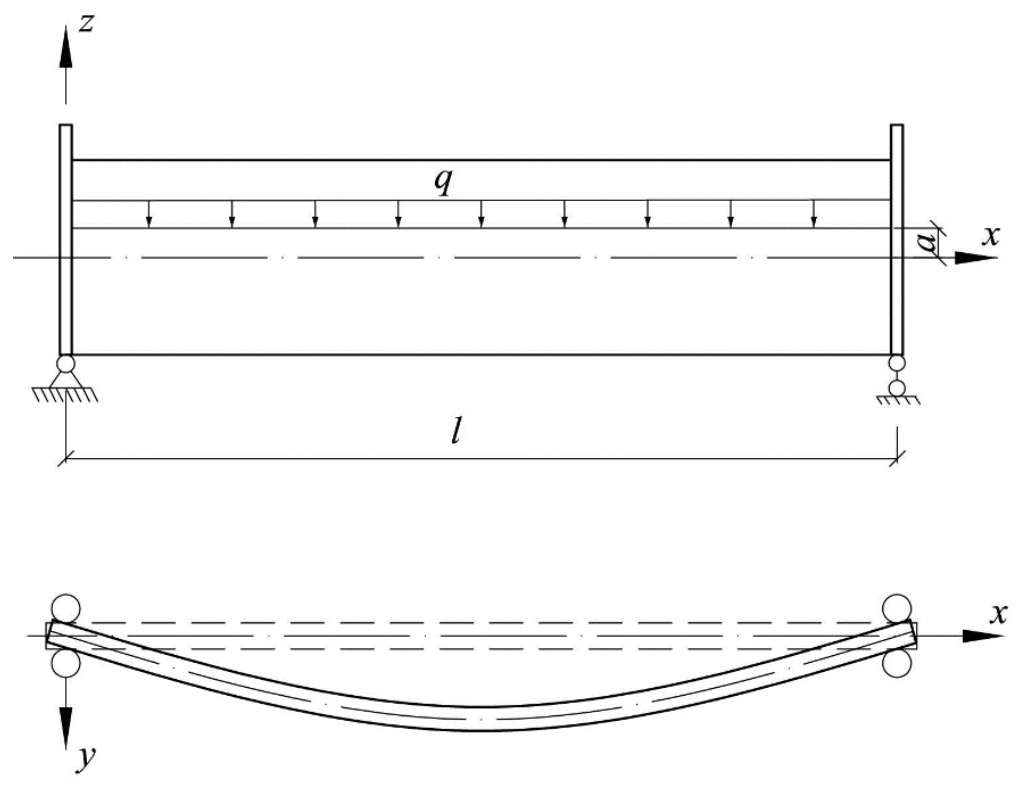

Fig. 1. Design scheme

We introduce the dimensionless coordinate $\xi=x / l$. The bending moment in the beam is determined by the formula:

$$
M_{y}=\frac{q x}{2}(l-x)=\frac{q l^{2}}{2} \xi(1-\xi)=q l^{2} \bar{M}(\xi) .
$$

We will find the critical load from the condition of minimum total energy $U$, which is defined as:

$$
U=W-A,
$$

where $W$ is the potential strain energy and $A$ is the external forces work.

The values of $W$ and $A$ for a beam with constant stiffness when a load is applied at the center of gravity of the cross section are determined by the formulas [3]:

$$
\begin{gathered}
W=\frac{1}{2}\left(E I_{z} \int_{0}^{l}\left(\frac{d^{2} v}{d x^{2}}\right)^{2} d x+G I_{t} \int_{0}^{l}\left(\frac{d \theta}{d x}\right)^{2} d x\right), \\
A=-\int_{0}^{l} M_{y} \theta \frac{d^{2} v}{d x^{2}} d x,
\end{gathered}
$$

where $v$ is the lateral deflection of the beam, $E I_{\mathrm{z}}$ is the flexural rigidity in the plane of least rigidity, $G I_{\mathrm{t}}$ is the torsional rigidity.

The second derivative of deflection is expressed through the twist angle as follows [4]:

$$
\frac{d^{2} v}{d x^{2}}=-\frac{M_{y} \theta}{E I_{z}} \text {. }
$$


Substituting (5) in (4), and then (4) and (3) in (2), we obtain:

$$
\begin{gathered}
U=\frac{1}{2}\left(G I_{t} \int_{0}^{l}\left(\frac{d \theta}{d x}\right)^{2} d x\right. \\
\left.-\frac{1}{E I_{z}} \int_{0}^{l} M_{y}^{2} \theta^{2} d x\right)=\frac{1}{2}\left(\frac{G I_{t}}{l} \int_{0}^{1}\left(\frac{d \theta}{d \xi}\right)^{2} d \xi-\frac{q^{2} l^{5}}{E I_{z}} \int_{0}^{l} \bar{M}^{2} \theta^{2} d \xi\right)= \\
=\frac{1}{2} \frac{G I_{t}}{l}\left(\int_{0}^{1}\left(\frac{d \theta}{d \xi}\right)^{2} d \xi-\lambda \int_{0}^{1} \bar{M}^{2} \theta^{2} d \xi\right),
\end{gathered}
$$

where $\lambda=\frac{q^{2} l^{6}}{G I_{t} E I_{z}}, \bar{M}(\xi)=\frac{M_{y}(\xi)}{q l^{2}}$.

The function of the twist angle is presented in the form of a series:

$$
\theta(\xi)=\sum_{i=1}^{n} a_{i} f_{i}
$$

where $a_{i}$ - undetermined coefficients, $f_{i}$ - basis functions that must satisfy the boundary conditions.

For the beam under consideration, the boundary conditions have the form:

$$
\theta(0)=\theta(1)=0
$$

Functions $f_{i}$ are accepted for these boundary conditions as:

$$
f_{i}=\sin \pi i \xi .
$$

The minimization of the functional $U$ is performed by the coefficients of the series (7):

$$
\frac{\partial U}{\partial a_{j}}=0, j=1 . . . n \rightarrow \frac{1}{2} \frac{G I_{t}}{l}\left(2 \sum_{i=1}^{n} a_{i} \int_{0}^{1} \frac{d f_{i}}{d \xi} \frac{d f_{j}}{d \xi} d \xi-2 \lambda \sum_{i=1}^{n} a_{i} \int_{0}^{1} \bar{M}^{2} f_{i} f_{j} d \xi\right)=0 .
$$

Expression (10) will be equivalent to the following matrix expression:

$$
([A]-\lambda[B])\{X\}=0,
$$

where $\{X\}$ is the vector of series coefficients, the elements of the matrices $[A]$ and $[B]$ are calculated by the formulas:

$$
A_{i j}=\int_{0}^{1} \frac{d f_{i}}{d \xi} \frac{d f_{j}}{d \xi} d \xi ; \quad B_{i j}=\int_{0}^{1} f_{i} f_{j} \bar{M}^{2}(\xi) d \xi
$$

After substituting (9) into (12) we obtain:

$$
A_{i j}=\left\{\begin{array}{c}
i j \pi^{2} / 2, i=j \\
0, i \neq j
\end{array}\right.
$$




$$
B_{i j}=\left\{\begin{array}{l}
-\frac{12 i j\left(i^{4}-j^{4}\right)\left[(-1)^{i+j}+1\right]}{\pi^{4}\left(i^{2}-j^{2}\right)^{5}}, i \neq j \\
\frac{\pi^{4} i^{4}+45}{240 i^{4} \pi^{4}}, i=j .
\end{array}\right.
$$

The system (11) has a nonzero solution if its determinant is equal to zero:

$$
|[A]-\lambda[B]|=0 .
$$

The critical load is determined from (11) by the formula:

$$
q_{c r}=\sqrt{\lambda_{\min }} \frac{\sqrt{G I_{t} E I_{z}}}{l^{3}}=K \frac{\sqrt{G I_{t} E I_{z}}}{l^{3}} .
$$

\section{Results and Discussion}

The table 1 shows the values of the coefficient $K$ obtained by our method for the beam under consideration for various numbers of members of the series. The results of the third and fourth approximations differ slightly.

Table 2. The dependence of the coefficient $K$ on the number of members of the series $n$.

\begin{tabular}{|l|c|c|c|c|c|}
\hline$n$ & 1 & 2 & 3 & 4 & 5 \\
\hline$K$ & 28.4624 & 28.4624 & 28.3151 & 28.3150 & 28.3150 \\
\hline
\end{tabular}

In paper [5], by directly integrating the differential stability equation, a value $K=28.3$ is obtained

The method also allows you to take into account the vertical displacement $a$ of the load relative to the center of gravity of the section.

In this case, an additional term $A_{1}$ appears in the expression for the work of external forces, having the form:

$$
A_{1}=\frac{q a}{2} \int_{0}^{l} \theta^{2} d x
$$

Expression (10), taking into account the additional term $A_{l}$, takes the form:

$$
\begin{gathered}
\frac{1}{2} \frac{G I_{t}}{l}\left(2 \sum_{i=1}^{n} a_{i} \int_{0}^{1} \frac{d f_{i}}{d \xi} \frac{d f_{j}}{d \xi} d \xi-2 \lambda \sum_{i=1}^{n} a_{i} \int_{0}^{1} \bar{M}^{2} f_{i} f_{j} d \xi-2 \sqrt{\lambda} \alpha \sum_{i=1}^{n} a_{i} \int_{0}^{1} f_{i} f_{j} d \xi\right)=0 \\
\text { where } \alpha=\frac{a}{l} \sqrt{\frac{E I_{z}}{G I_{t}}}
\end{gathered}
$$

Using expression (12) leads to the equation: 


$$
|[A]-\lambda[B]-\sqrt{\alpha} \lambda[C]|=0 .
$$

The method for solving equation (20) is presented in [6].

The coefficients of the matrix $[C]$ are determined by the formulas:

$$
C_{i j}=\int_{0}^{1} f_{i} f_{j} d \xi .
$$

For the basis functions (9), the matrix $[C]$ is equal to half the unit matrix $[E]$. The critical load, as before, is determined by the formula (17).

Figure 2 shows the graph of the dependence of the coefficient $K$ on the $\alpha$ parameter. The dashed line corresponds to the simplified linear dependence proposed in [6] on the basis of solution by the finite difference method:

$$
K=28.675-40.317 \alpha \text {. }
$$

There is a very good agreement between the results, which indicates their reliability.

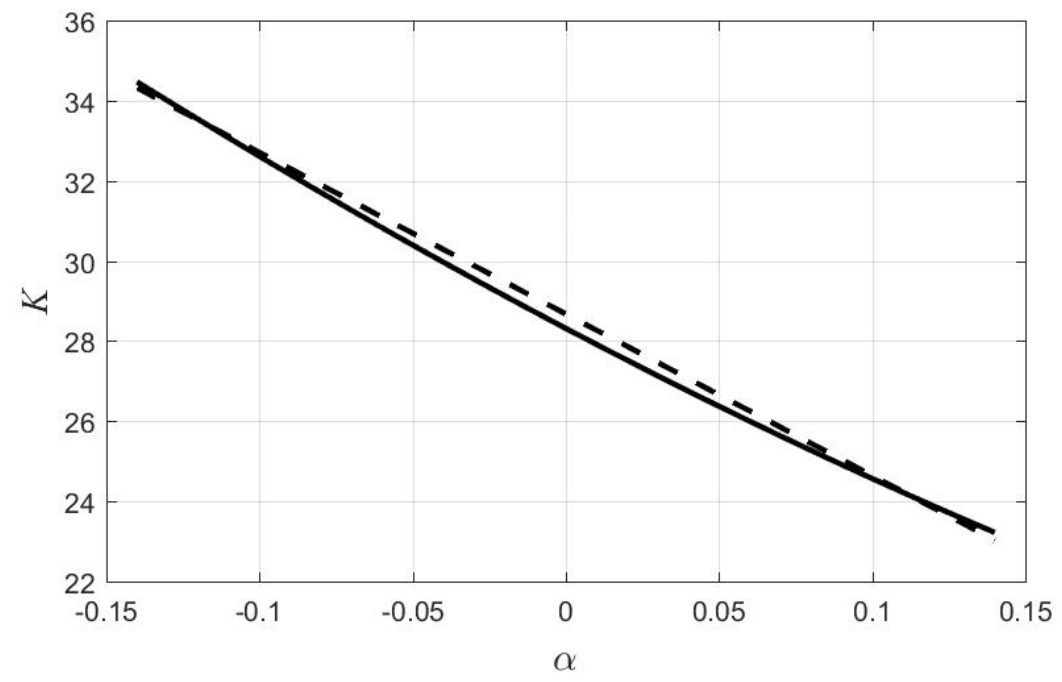

Fig. 2. The dependence of the coefficient $\mathrm{K}$ on the parameter $\alpha$

\section{Conclusions}

The proposed version of the energy method makes it possible to obtain solutions under arbitrary boundary conditions and loads. The number of members in a series can be unlimited. The energy method is also very effective for beams of variable stiffness. Compared to the direct solution of the differential equation, which is used, for example in [7-10], in the case of a piecewise linear change in stiffness, there is no need to satisfy the boundary conditions at the ends of each section.

\section{References}

1. S. P. Timoshenko, J. M. Gere, Theory of elastic stability (McGraw-Hill, 1961) 
2. S. Yazyev et al., E3S Web of Conferences. 97, 04066 (2019)

3. V.M. Makushin, N.G. Savel'yev, N. V. Khung. in: Raschety na prochnost. 14, 268289 (1969)

4. A.A. Karamisheva, S.B. Yazyev, A.A. Avakov, Procedia Engineering. 150, 1872-1877 (2016)

5. A. S. Volmir, Stability of deformable systems (Nauka, 1967)

6. A. S. Chepurnenko, V.V. Ulianskaya, S.B. Yazyev, I.M. Zotov, MATEC Web of Conferences. 196, 01003 (2018)

7. A. P. Lapina, I.M. Zotov, A.S. Chepurnenko, B.M. Yazyev, E3S Web of Conferences. 97, 04067 (2019)

8. A.F. Smirnov et al., Structural mechanics. Dynamics and stability of structures (Stroizdat, 1984)

9. I.M. Zotov, A.P. Lapina, A.S. Chepurnenko, B.M. Yazyev, Materials Science Forum. 974, 551-555 (2019)

10. I.M. Zotov, A.P. Lapina, A.S. Chepurnenko, B.M. Yazyev, IOP Conference Series: Materials Science and Engineering. 661 (2019) 